\title{
Optimal Graph Based Segmentation Using Flow Lines with Application to Airway Wall Segmentation
}

\author{
Jens Petersen ${ }^{1}$, Mads Nielsen ${ }^{1}$, Pechin Lo $^{1}$, Zaigham Saghir ${ }^{2}$, Asger Dirksen ${ }^{2}$, \\ and Marleen de Bruijne ${ }^{1,3}$ \\ 1 Department of Computer Science, University of Copenhagen, Denmark \\ 2 Department of Respiratory Medicine, Gentofte Hospital, Denmark \\ 3 Biomedical Imaging Group Rotterdam, Departments of Radiology \& Medical \\ Informatics, Erasmus MC, Rotterdam, The Netherlands
}

\begin{abstract}
This paper introduces a novel optimal graph construction method that is applicable to multi-dimensional, multi-surface segmentation problems. Such problems are often solved by refining an initial coarse surface within the space given by graph columns. Conventional columns are not well suited for surfaces with high curvature or complex shapes but the proposed columns, based on properly generated flow lines, which are non-intersecting, guarantee solutions that do not self-intersect and are better able to handle such surfaces.

The method is applied to segment human airway walls in computed tomography images. Comparison with manual annotations on 649 crosssectional images from 15 different subjects shows significantly smaller contour distances and larger area of overlap than are obtained with recently published graph based methods.

Airway abnormality measurements obtained with the method on 480 scan pairs from a lung cancer screening trial are reproducible and correlate significantly with lung function.
\end{abstract}

Keywords: Segmentation, graph, flow lines, airway walls

\section{Introduction}

Graph based methods similar to Wu and Chen's [10] polynomial time solutions to optimal net surface problems using minimum cut algorithms have seen a growing use within medical image segmentation in the last couple of years. The methods can find the globally optimal solution for multiple surfaces in multiple dimensions given surface cost functions, and various geometric constraints $[4,5$, $9,11]$.

In order to use these methods, the segmentation problem needs to be transformed from image space to some discretized graph space defined by a set of columns. Each column is associated with a point on the sought surface and represents the set of possible solutions, or positions, it can take. The suitability of the graph space depends on how well plausible solutions in image space can be 
represented. For instance if a graph column does not cross the sought surface, then this surface can not be represented in graph space. Similarly, admissible solutions in graph space should represent valid surfaces in image space. If this is not the case the found solutions can for instance have self-intersections. It is also important that the transformation allows for a meaningful representation of the surface cost functions and geometric constraints, such as for instance surface smoothness.

There have been methods using simple mathematical transformations, such as $[4,9]$, in which images of tubular airway segments were unfolded using polar transforms in two or three-dimensions. The graph columns were oriented perpendicular to the resulting straight contours or terrain like surfaces allowing for an easy representation of surface smoothness constraints. However such approaches only work with very simple shapes.

In [5] a rough initial segmentation was used to define the transform. The columns were placed at surface points and oriented along the surface normals. By limiting their length to the minimum distance to the medial axes of the initial segmentation, problems with intersecting columns and thus possible selfintersecting surfaces were avoided. The approach can however result in columns that are too short to reach the desired solution, as shown in Fig. 1(a). In such areas the curvature of the surfaces through the columns also often does not follow the curvature of the sought surfaces, which can make it harder to construct good segmentation cost functions.

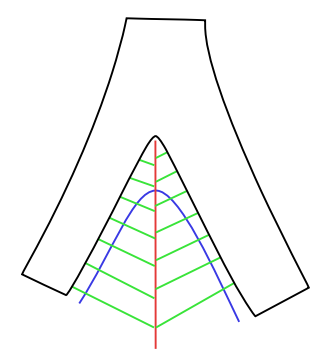

(a) Medial Axes

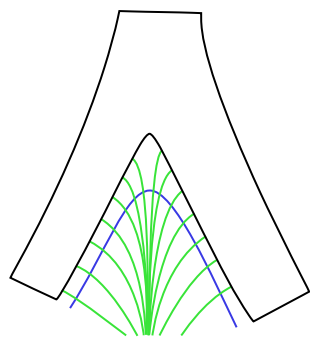

(b) Flow Lines

Fig. 1. Figure 1(a) illustrates the fishbone like structure of surface normal direction columns (green) based on the distance to the medial axis (red) in areas of the initial segmentation (black) with high curvature. Notice that the four inner-most columns do not cross the sought surface border (blue), which means that the segmented surface will be wrong in these positions. Figure 1(b) shows the advantage of columns based on flow lines (green). Notice that all columns cross the sought surface, and the curvature of the surfaces through the columns are more similar to the curvature of the sought surface.

Yin et al. [11] suggested columns inspired by the non-intersecting property of electric lines of force. The columns were constructed by placing electrical charges at surface points of the initial segmentation and tracing the electric lines of force 
within the field inward and outward. The method is computationally intractable for large scale problems, as every surface point charge influences the computation of every electric line of force. Furthermore the electric lines of force can behave erratically if the initial segmentation contains small scale errors or noise.

We suggest to combine a regularization of the initial segmentation with a curvilinear transformation using an implementation which is computationally tractable for large scale problems. The columns are defined by greatest ascent and descent flow lines, calculated from the convolution of the initial segmentation with a $C^{1}$ continuous integrable filter. Such flow lines are uniquely defined and also non-intersecting, and fast approximations can often be computed by limiting the extent of the convolution kernels. Furthermore, regularization terms are naturally incorporated into the framework, making it easier to deal with noise and small errors in the initial segmentation. Figure 1(b) illustrates the concept.

We apply the method to the problem of segmenting the human airway walls in three dimensions in computed tomography images, to allow quantification of abnormalities such as airway thickening and bronchiectasis. This is a dual surface problem, consisting of an inner and an outer surface boundary, between the airway lumen and the wall and between the lung parenchyma and the wall respectively, where the many bifurcations form regions of high curvature that would cause problems for conventional graph construction approaches.

\section{Method}

\subsection{Initial Segmentation}

We will assume the existence of a coarse initial segmentation, a single object given by the voxels in the set $S$, whose surface should be roughly similar to the surfaces we are looking for. For our application we used the airway tree segmentation algorithm described in [7], which returns a binary three-dimensional segmentation of the airway lumen.

The initial segmentation needs to be converted to a mesh. To this end, we used vertices at the center of each surface face and the neighborhood given by the face edge neighbors, such that each vertex has 4 neighbors. We will denote the vertices in this mesh with $V_{B}$, and represent the neighborhood with an edge set $E_{B}$, where $(i, j) \in E_{B}$ denotes that the vertices $i, j \in V_{B}$ are neighbors. The resolution of the mesh used in the experiments was $0.5 \mathrm{~mm} \times 0.5 \mathrm{~mm} \times 0.5 \mathrm{~mm}$.

\subsection{Flow Lines}

A flow line in a vector field is tangent to the field at each point, and if it is defined in terms of some scalar potential, it will follow the direction with the greatest rate of change of this potential. Consider the potentials $E$ defined by the convolution:

$$
E(\mathbf{x})=\int Q(\hat{\mathbf{x}}) R(\hat{\mathbf{x}}-\mathbf{x}) d \hat{\mathbf{x}}
$$


where $\mathbf{x}$ is the position to be evaluated and $Q$ is an indicator function for the initial segmentation:

$$
Q(\mathbf{x})=\left\{\begin{array}{l}
1 \text { if } \mathbf{x} \in S \\
0 \text { if } \mathbf{x} \notin S
\end{array} .\right.
$$

In this work we experimented with generating the potentials from two different types of filters. The first are of the form:

$$
R(\mathbf{x})=\frac{1}{\alpha+|\mathbf{x}|^{2}}
$$

where $\alpha>0$ is a regularization constant, which makes $R$ well defined for all $\mathbf{x}$. Increasing the value of $\alpha$ has the added effect of smoothing the result, which is useful if the initial segmentation contains a lot of noise. Notice that when $\alpha \rightarrow 0, E$ becomes equal to the electric potential times a constant arising from a 'charge density' given by $Q$. This option therefore is similar to the method introduced in [11], but rather than having a discrete set of surface point 'charges', which introduce local singularities, it is defined everywhere and thus allows us to trace the flow lines consistently through the surface. We will refer to this as the potential kernel/filter.

The second type of flow line is given by the Gaussian kernel, that is:

$$
R(\mathbf{x})=c e^{-|\mathbf{x}|^{2} /\left(2 \sigma^{2}\right)}
$$

which is separable, unlike the potential filter. The convolution operation thus becomes much less expensive.

The flow lines are traced inward in the gradient direction and outward in the negative gradient direction, sampling the columns at regular arc length intervals. This was done using a sampling interval of $0.5 \mathrm{~mm}$. At some point the gradient flattens to the point where we can no longer trace the column due to numerical issues or due to the size of the convolution kernel, resulting in a column with a finite number of inner and outer column points.

\subsection{Graph}

We use an optimal graph construction technique similar to Ishikawa's [3], which has slightly fewer edges than the $\mathrm{Wu}$ and Chen method [10]. It should be noted that neither of these papers deal with columns of varying lengths, however this can easily be dealt with similar to how the out of bounds edges were handled by Ishikawa [3], see (7) and Fig. 2(b).

We have a column $V_{i}$ traced from each mesh vertex $i \in V_{B}$, with the following set of points: $\left\{i_{-I_{i}}, i_{-\left(I_{i}-1\right)}, \ldots, i_{0}, \ldots, i_{O_{i}}\right\}$, where $I_{i}$ and $O_{i}$ correspond to the number of inner and outer column points relative to $i_{0}=i$, and need to construct a maximum flow graph $G=(V, E)$, with vertices $V$ and edges $E$, to find the set of surfaces $M$. We thus construct columns of vertices $V_{i}^{m}=\left\{i_{k}^{m} \mid i_{k} \in V_{i}\right\}$ in $V$, where $m \in M$ such that:

$$
V=\bigcup_{i \in V_{B}, m \in M} V_{i}^{m} \cup\{s, t\} .
$$


Here $s$ and $t$ denote the source and sink nodes respectively. In the case of airway wall segmentation, $M=\{0,1\}$ would denote the fact that there is an inner and outer surface sub-graph.

Let $(v \stackrel{c}{\rightarrow} u)$ denote a directed edge from vertex $v$ to vertex $u$ with capacity $c$. We define a cost function $w\left(i_{k}^{m}\right) \geq 0$, mapping a node with index $k \in\left\{-I_{i}, 1-\right.$ $\left.I_{i}, \ldots, 0, \ldots, O_{i}\right\}$ in a column $V_{i}$ to to the inverse likelihood that it is part of the surface $m$, see Sect. 2.4. Such a data term can be implemented by the following edges:

$$
\begin{aligned}
E_{\text {data }}=\{ & \left\{\left(i_{k}^{m} \stackrel{w\left(i_{k}^{m}\right)}{\rightarrow} i_{k+1}^{m}\right) \mid i_{k}^{m}, i_{k+1}^{m} \in V_{i}^{m}\right\} \cup \\
& \left.\left\{\left(i_{O_{i}}^{m} \stackrel{w\left(i_{O_{i}}^{m}\right)}{\rightarrow} t\right),\left(s \stackrel{\infty}{\rightarrow} i_{I_{i}}^{m}\right)\right\} \mid i \in V_{B}, m \in M\right\} .
\end{aligned}
$$

Since each column is a direct line of flow from the source to the sink, it must be cut at least once. However in some degenerate cases, multiple cuts might exist in each column. This is something to be avoided, as it could change the topology of the surface. A solution is to add infinite cost edges opposite to the data term edges:

$$
E_{\infty}=\left\{\left(i_{k}^{m} \stackrel{\infty}{\rightarrow} i_{k-1}^{m}\right) \mid i \in V_{B}, m \in M, i_{k-1}^{m}, i_{k}^{m} \in V_{i}^{m}\right\}
$$

An example of these edges is given in Fig. 2(a).

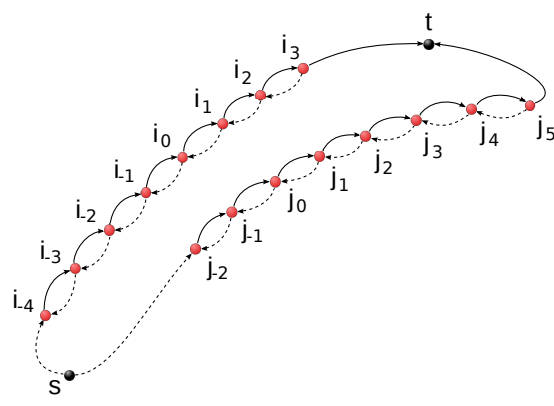

(a) Intra column edges

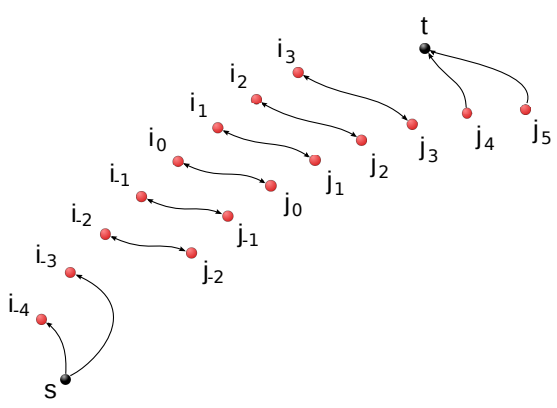

(b) Inter column edges

Fig. 2. Two example neighboring columns $V_{i}$ and $V_{j}$, with $I_{i}=4$ and $I_{j}=2$ inner column points and $O_{i}=3$ and $O_{j}=5$ outer column points (note $m$ superscript left out for clarity). The data term edges are given by the black arrows and the infinite cost edges are dotted in Fig. 2(a). The inter column edges generated from a linear edge penalty function in Fig. 2(b) in black. If the edge penalty is of the form: $f_{i, j}(x)=p^{m} x$, the (bi-)directional capacity of these inter column edges are then given by $p^{m}$. Notice that when combining the inter and intra column edges the vertex $j_{5}$ has two edges connecting it to the sink node. This is just for illustrative purposes, in practice the capacities of these are summed and implemented with a single edge. The inter column edge from the $s$ to $i_{-4}$ can be left out completely. 
Let $f_{i, j, m, n}(|k-l|)$ define a convex non-decreasing function describing the inverse likelihood that both vertex $i_{k} \in V_{i}$ and $j_{l} \in V_{j}$ are part of the surfaces $m, n \in M$ respectively. Note $m$ can be equal to $n$ and $i$ can be equal to $j$, but not simultaneously. This can be thought of as an edge penalty, since it attributes a penalty of including the 'edge' $\left(i_{k}^{m}, j_{l}^{n}\right)$ in the solution and can be used to implement surface smoothness and separation penalties, see (9). Both Ishikawa [3] and $\mathrm{Wu}$ and Chen [10] show how such edge penalties can be implemented in the case where the columns have equal length. The following inter column edges extend the implementation to the case where both the number of inner and outer column points can vary:

$$
\begin{aligned}
E_{\text {inter }}=\{ & \left\{\left(i_{k}^{m} \stackrel{\triangle_{i, j, m, n}(k-l)}{\rightarrow} j_{l}^{n}\right) \mid i_{k}^{m} \in V_{i}^{m}, j_{l}^{n} \in V_{j}^{n}\right\} \cup \\
& \left\{\left(s \stackrel{\triangle_{i, j, m, n}(k-l)}{\rightarrow} j_{l}^{n}\right) \mid j_{l}^{n} \in V_{j}^{n}, k \in L(j, i)\right\} \cup \\
& \left\{\left(i_{k}^{m} \stackrel{\triangle_{i, j, m, n}(k-l)}{\rightarrow} t\right) \mid i_{k}^{m} \in V_{i}^{m}, l \in U(i, j)\right\} \\
& \left.\mid i, j \in V_{B}, m, n \in M\right\},
\end{aligned}
$$

where $L$ and $U$ describe the needed edge endpoints that are out of bounds:

$$
\begin{aligned}
L(i, j) & =\left\{k \mid I_{i}>I_{j}, k \in\left\{-I_{i}, 1-I_{i}, \ldots,-I_{j}-1\right\}\right\} \\
U(i, j) & =\left\{k \mid O_{i}>O_{j}, k \in\left\{O_{i}, O_{i}-1, \ldots, O_{j}+1\right\}\right\},
\end{aligned}
$$

and $\triangle$ the capacity of the edges calculated from the edge penalty function:

$$
\triangle_{i, j, m, n}(x)=\left\{\begin{array}{ll}
0 & \text { if } x<0 \\
f_{i, j, m, n}(1)-f_{i, j, m, n}(0) & \text { if } x=0 \\
\left(f_{i, j, m, n}(x+1)-f_{i, j, m, n}(x)\right)- & \text { if } x>0 \\
\left(f_{i, j, m, n}(x)-f_{i, j, m, n}(x-1)\right) &
\end{array} .\right.
$$

We used the following edge penalty functions in our experiments:

$$
f_{i, j, m, n}(x)=\left\{\begin{array}{ll}
p^{m} x & \text { if } m=n \text { and }(i, j) \in E_{B} \\
q x & \text { if } m \neq n \text { and } i=j \\
0 & \text { else }
\end{array} .\right.
$$

$p^{m}$ is the smoothness penalty, defining the cost of each index the solution varies between neighboring columns in the same surface $m . q$ is the separation penalty, defining the cost inherent in the solution for each index the surfaces are separated in each column. The advantage of using linear edge penalty functions is that far fewer edges are needed to implement the penalty as $\triangle_{i, j, m, n}(x)=0$ for all $x \neq 0$. An illustration of these edges is given in Fig. 2(b). The total edge set $E$ in the maximum flow graph is given by:

$$
E=E_{\text {data }} \cup E_{\infty} \cup E_{\text {inter }} .
$$

Note the edge penalties described in this Sect. are soft. It is possible to add hard constraints as well, by adding infinite cost edges between the columns. We refer to $[4,10]$ for examples of this.

We used the algorithm described in [1] to find the minimum cut. 


\subsection{Cost Functions}

In the case of airway walls, the columns will usually start inside the air-filled lumen area, which has low density, move through the airway wall where the density rises, and finally end up in the lung parenchyma where the density falls again. The CT intensity directly reflects this density change. A common way to find such boundaries is to use weightings of the first and second order derivatives of the intensity along the columns $[4,5,9]$. In this way the positions of the found surfaces can be adjusted relative to the 'real' surfaces. For the experiments described in this paper we used the cost functions of Petersen et al. [9], with inner and outer surface derivative weights given by $\gamma^{0}, \gamma^{1} \in[0,1]$. The derivatives are obtained using central differences from cubic interpolated values.

\section{Experiments and Results}

\subsection{Data}

The material used comes from the Danish lung cancer screening trial [8]. The images were obtained using a Multi Detector CT scanner with a low dose (120 $\mathrm{kV}$ and $40 \mathrm{mAs}$ ), reconstructed using a hard kernel with a resolution of approximately $0.78 \mathrm{~mm} \times 0.78 \mathrm{~mm} \times 1 \mathrm{~mm}$.

For evaluation, we used manual annotations in 649 two-dimensional crosssectional images with a resolution of $0.5 \mathrm{~mm} \times 0.5 \mathrm{~mm}$ extracted at random positions perpendicular to and centered on the airways in 15 scans. Some of these images contain more than one airway branch. Such other branches were marked and excluded from the analysis. The images were split randomly in a training and a test data set, consisting of 329 and 319 images from 8 and 7 subjects.

To evaluate suitability of the segmentations for deriving measures of airway morphology as a sign of Chronic Obstructive Pulmonary Disease (COPD), 480 subjects for whom repeated scans and spirometry within a two year period were available, were randomly selected to evaluate reproducibility of measures of airway morphology as well as their correlation with lung function.

\subsection{Comparison to Existing Approaches}

Experiments were conducted with a recently published two-dimensional method [9] and three three-dimensional methods based on different ways of constructing the columns. One was a method using straight columns $S_{k, \tau}$, as described in [5], the medial axes and normals were determined using the method of reference [2] using $k$ neighbors and an error tolerance of $\tau$ respectively. We refer to the original article for a definition of these parameters. Normals with too large errors were replaced by a nearest neighbor normal. The other two methods used the proposed flow line columns calculated from the potential and Gaussian kernels, denoted $P_{\alpha}$ and $G_{\sigma}$ respectively. 
Let $M_{l}, M_{a}$ and $X$ denote the manually marked lumen, airway and excluded parts respectively. Similarly let $A_{l}$ and $A_{a}$ denote the parts segmented by the algorithms. We then evaluate the correctness of a segmentation in the crosssections using the relative area of overlap outside the excluded area, $\Phi$ as follows:

$$
\Phi\left(M_{l}, M_{a}, A_{l}, A_{a}, X\right)=\frac{\left|\left(M_{l} \cap A_{l}\right) / X\right|}{\left|M_{l} / X\right|+\left|A_{l} / X\right|}+\frac{\left|\left(M_{a} \cap A_{a}\right) / X\right|}{\left|M_{a} / X\right|+\left|A_{a} / X\right|} .
$$

Define the contour $\mathcal{C}(A)$ of an area $A$ as the set of pixels belonging to $A$, where at least one of the pixels in the standard 4-neighborhood is not part of $A$. The average contour distance was then defined by:

$$
\Psi\left(M_{l}, M_{a}, A_{l}, A_{a}, X\right)=\operatorname{mean}_{x \in \mathcal{C}\left(A_{l} / X\right)} d\left(\mathcal{C}\left(M_{l} / X\right), x\right),
$$

where $d(A, x)$ defines the minimum euclidean distance between the point $x$ and the set $A$.

Parameter Tuning. The methods have inner and outer smoothness penalties, inner and outer cost function derivative weightings and separation penalties, denoted $p^{m}, \gamma^{m}$ and $q$ where $m \in M$ respectively. Optimal values for these parameters for each of the methods were obtained by searching the parameter space on the training data set using an iterative algorithm. In each iteration a parameter is searched by probing left and right search interval limits. If no improvement in the mean value of $\Phi$ is detected the intervals are halved around the current best guess and the process is repeated. This continues until a better guess is found or the difference between the left and right search intervals becomes less than some threshold. The search then proceeds with the next parameter in a loop with reset left and right search interval limits until no more parameters get updated. In order to avoid getting stuck in a local minima, the algorithm was repeated a number of times using random values as initial guesses for the parameters.

Since parameter optimization is time consuming, a small set of pilot experiments were performed on the training set to determine suitable values of $k, \tau, \alpha$ and $\sigma$. The optimal value of $\tau$ was found to be about 2.5 , meaning roughly $3 \%$ of the normals were discarded for having an error above the tolerance. The segmentation results improved with increasing $k$, flattening out at about 64 . $k$ is inversely related to the resolution of the medial axis and thus directly related to the length of the columns and number of self-intersections. The fact that the relative high value of 64 works best seems to indicate that the straight column method suffers from too short columns. The experiments with $\alpha$ indicated that the potential kernel did not require additional regularization, so we chose a value of $\alpha=10^{-4} \mathrm{~mm}$ as this would have practically no regularizing effect, yet still allowed the filter to be well defined everywhere. A similar conclusion holds for $\sigma$, however as the Gaussian tends to zero much faster than the potential kernel, the choice of $\sigma$ seems to be a trade-off between too short columns or too much regularization. A value of $0.45 \mathrm{~mm}$ provided the best results for our case. 
Results. Running the proposed method on an image from our data usually takes less than 10 minutes (Intel Xeon $1.60 \mathrm{GHz}$ using no parallelization). Figure 3 shows a segmentation result in three dimensions and cross-sections illustrating results of $G_{\sigma=0.45}$ and $S_{k=64, \tau=2.5}$.

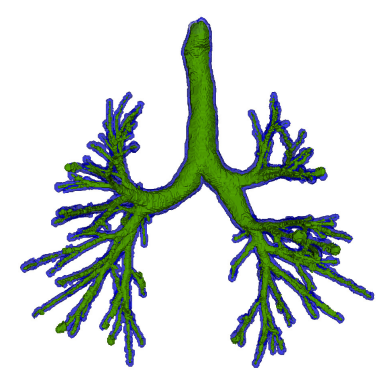

(a)

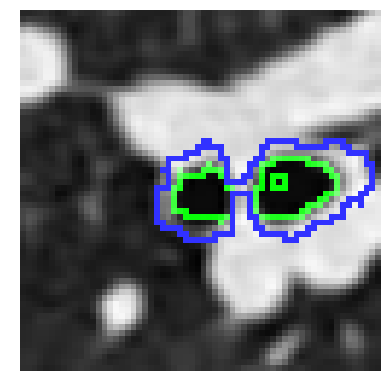

(b)

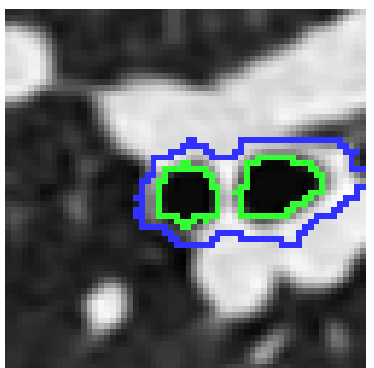

(c)

Fig. 3. Figure 3(a), 3(b) and 3(c) show the inner (green) and outer (blue) airway wall surface of a segmentation result in three dimensions obtained using $G_{\sigma=0.45}$ and two cross-sections near a bifurcation obtained with $S_{k=64, \tau=2.5}$ and $G_{\sigma=0.45}$ respectively. Notice how the outer surface cannot be correctly found using $S_{k=64, \tau=2.5}$ in the region with high curvature between the airway branches and there is even a hole in the segmentation, probably due to self-intersecting surfaces.

Table 1 shows the results of the comparisons with the manual annotations in the test data set, for each of the investigated methods using the optimal parameters. $G_{\sigma=0.45}$ achieved the best result when measured with both metrics, whereas $S_{k=64, \tau=2.5}$ and $2 D$ were the worst in terms of the relative area of overlap and average contour distance respectively. $G_{\sigma=0.45}$ was significantly better than any of the other three-dimensional methods and also significantly better than $2 D$ using $\Psi(p<0.05)$ and shows the smallest variance in the quality of the results of all the methods $(p<0.0001)$. Results were compared using a paired-sample $t$-test and a two-sample $F$-test respectively.

Table 1. The results of different methods and kernels on the test data set. Mean \pm standard deviation of (11) and (12). The best result marked with a bold font.

\begin{tabular}{lllll}
\hline & $2 D$ & $S_{k=64, \tau=2.5}$ & $P_{\alpha=10^{-4}}$ & $G_{\sigma=0.45}$ \\
\hline$\Phi$ & $0.884 \pm 0.079$ & $0.865 \pm 0.086$ & $0.880 \pm 0.079$ & $\mathbf{0 . 8 9 0} \pm \mathbf{0 . 0 5 9}$ \\
$\Psi(\mathrm{mm})$ & $0.115 \pm 0.176$ & $0.105 \pm 0.132$ & $0.113 \pm 0.177$ & $\mathbf{0 . 0 9 2} \pm \mathbf{0 . 1 0 2}$ \\
\hline
\end{tabular}




\subsection{Tree Extraction and Measurements}

Airway centerlines and branch generations were extracted from the airway tree with a front propagation method, as described in [6]. Airway morphology was quantified using the Inner Volume $(I V)$ and the Wall Volume Percentage $(W V \%)$, which are three-dimensional extensions to commonly used measures of airway abnormality [9]. The segmentations were grouped in generations by assigning each voxel to the generation of the nearest centerline point. Let $W$ denote the area classified as belonging to a specific generation, then the measures were computed in individual generations as follows:

$$
\begin{aligned}
& I V=\left|W \cap A_{l}\right|, \\
& W V \%=100 \times W V /(I V+W V) \%,
\end{aligned}
$$

where $W V=\left|W \cap A_{a} / A_{l}\right|$. Branches of generation 1 to 8 were included in the measurements. The trachea, defined as generation 0 , was excluded.

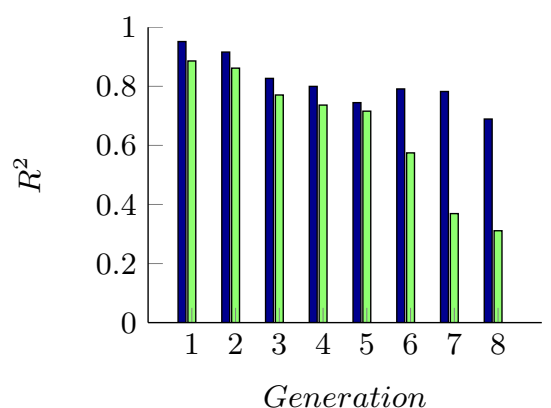

(a) Reproducibility

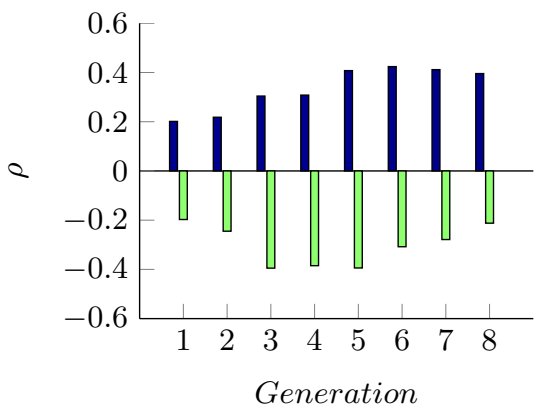

(b) Correlation with Lung Function

Fig. 4. Figure 4(a) shows the reproducibility of the measures, $I V$ (blue) and $W V \%$ (green), in repeated scans quantified as $R^{2}$ in branch generations 1 to 8. Figure 4(b) shows statistically significant correlation with lung function in the same generations of the same measures.

The change in the measures during the roughly one year period between the repeated scans is assumed to be mostly due to measurement variability. This is a fair assumption given the relatively slow development of COPD and was backed up by paired-sample $t$-tests, showing no significant difference in the means of the measures in the same generation in repeated scans using a Bonferroni adjusted significance level of $p=0.05$. It is thus possible to quantify the reproducibility of the measures, with the coefficient of determination, calculated from Pearson product moment correlation coefficients of the measures in repeated scans, see Fig. 4(a).

$I V$ is the most reproducible measurement, which is to be expected given the high contrast ratio between the air-filled lumen and high density wall. The 
reproducibility of $W V \%$, which depend on the segmentation of the outer surface, show however that it is also determined with enough precision to allow reproducible measurements, up to roughly generation 6 .

Figure 4(b) shows Spearman's correlation coefficients $\rho$ of the measures and lung function measured by Forced Expiratory Volume in one second as a percentage of the predicted value (FEV1 (\% predicted)). $I V$ is positively correlated, indicating luminal narrowing with COPD, whereas $W V \%$ is negatively correlated, indicating wall thickening, which is in agreement with current knowledge of the disease process in COPD. These results indicate the method can be used to measure abnormalities caused by COPD up to at least generation 8 .

\section{Discussion and Conclusion}

In this paper we performed extensive parameter tuning for all methods to allow for an as objective comparison as possible. However, it is our experience that results are not very sensitive to the settings of these parameters and suitable settings can be obtained by something as simple as a few trial and error runs using manual inspection of the segmentation results.

The results indicate the importance of choosing a good convolution kernel. For our specific application the Gaussian performed better than the potential, which we think is mainly due to it tending to zero much faster, limiting long range effects. More experiments will be needed to investigate which kernels are suitable to which applications.

The proposed segmentation method is not able to extend the initial segmentation beyond the lengths of the graph columns, and is thus not able to make up for larger errors, such as missing branches. This can explain a large part of the decline in reproducibility with generations, seen in Fig. 4(a). For instance investigations on the amount of segmented branches compared to the theoretical maximum, assuming a binary tree structure, revealed that almost all the branches were segmented in generations 5 , whereas the number had dropped to about $50 \%$ in generation 6 . Measurements conducted in corresponding branches, as opposed to generations, might thus still be reproducible after generation 6 and 8.

To conclude, a new graph construction technique applicable to multi-dimensional multi-surface segmentation problems was proposed. The method runs in polynomial time and is able to penalize for non-smoothness and separation of the found surfaces. The results are guaranteed to not self-intersect and are robust in regions with large curvature.

We applied the method to the problem of segmenting human airway walls in $\mathrm{CT}$ images and results were shown to be significantly more accurate than those of recently published two- and three-dimensional methods. Large scale evaluations on 480 images from a lung cancer screening trial, showed good reproducibility of the obtained airway abnormality measures and a significant correlation with lung function. 
Acknowledgements This work is partly funded by the Netherlands Organisation for Scientific Research (NWO), and AstraZeneca, Sweden.

\section{References}

1. Boykov, Y., Kolmogorov, V.: An Experimental Comparison of Min-cut/Max- Flow Algorithms for Energy Minimization in Vision. IEEE Trans. Pattern Anal. Mach. Intell. 26(9), 1124-1137 (2004)

2. Dey, T.K., Sun, J.: Normal and Feature Estimation from Noisy Point Clouds. In: Proceedings of the 26th International Conference on Foundations of Software Technology and Theoretical Computer Science. pp. 21-32 (2006)

3. Ishikawa, H.: Exact Optimization for Markov Random Fields with Convex Priors. IEEE Trans. Pattern Anal. Mach. Intell. 25(10), 1333-1336 (October 2003)

4. Li, K., Wu, X., Chen, D.Z., Sonka, M.: Optimal Surface Segmentation in Volumetric Imagesi - A Graph-Theoretic Approach. IEEE Trans. Pattern Anal. Mach. Intell. 28(1), 119-134 (2006)

5. Liu, X., Chen, D.Z., Wu, X., Sonka, M.: Optimal Graph-Based Segmentation of 3D Pulmonary Airway and Vascular Trees Across Bifurcations. In: First International Workshop on Pulmonary Image Analysis. pp. 103-111 (2008)

6. Lo, P., van Ginneken, B., Reinhardt, J.M., de Bruijne, M.: Extraction of Airways from CT (EXACT'09). In: The Second International Workshop on Pulmonary Image Analysis. pp. 175-189 (2009)

7. Lo, P., Sporring, J., Pedersen, J.J.H., de Bruijne, M.: Airway Tree Extraction with Locally Optimal Paths. In: Yang, G.Z., Hawkes, D., Rueckert, D., Noble, A., Taylor, C. (eds.) Medical Image Computing and Computer-Assisted Intervention MICCAI 2009, LNCS, vol. 5762, pp. 51-58. Springer (2009)

8. Pedersen, J.H., Ashraf, H., Dirksen, A., Bach, K., Hansen, H., Toennesen, P., Thorsen, H., Brodersen, J., Skov, B.G., Døssing, M., Mortensen, J., Richter, K., Clementsen, P., Seersholm, N.: The danish randomized lung cancer CT screening trial-overall design and results of the prevalence round. J Thorac Oncol 4(5), 608614 (May 2009)

9. Petersen, J., Lo, P., Nielsen, M., , Edula, G., Ashraf, H., Dirksen, A., de Bruijne, M.: Quantitative Analysis of Airway Abnormalities in CT. In: Karssemeijer, N., Summers, R. (eds.) Medical Imaging 2010: Computer-Aided Diagnosis. Proceedings of SPIE. vol. 7624 (2010)

10. Wu, X., Chen, D.: Optimal Net Surface Problems with Applications. In: Widmayer, P., Eidenbenz, S., Triguero, F., Morales, R., Conejo, R., Hennessy, M. (eds.) Automata, Languages and Programming, LNCS, vol. 2380, pp. 775-775. Springer (2002)

11. Yin, Y., Song, Q., Sonka, M.: Electric Field Theory Motivated Graph Construction for Optimal Medical Image Segmentation. In: Torsello, A., Escolano, F., Brun, L. (eds.) Graph-Based Representations in Pattern Recognition, LNCS, vol. 5534, pp. 334-342. Springer (2009) 